\title{
Neutrinos from Relativistic Outflows of Fast Spinning Magnetars
}

\author{
Qinghuan Luo ${ }^{\mathrm{A}}$ \\ A School of Physics, University of Sydney, Sydney NSW 2006, Australia. \\ Email: luo@physics.usyd.edu.au
}

Received 2004 September 14, accepted 2005 February 2

\begin{abstract}
Pulsars may be born with a short rotation period of milliseconds with the magnetic field amplified through dynamo processes up to $\sim 10^{15}-10^{16} \mathrm{G}$. Such millisecond magnetars spin down rapidly, emitting bursts of high-energy neutrinos and gamma rays. Specifically, acceleration of ions in both the polar gap (as in a normal pulsar) and the relativistic magnetar wind is considered. In both cases ions can be accelerated to ultra-high energies and these energetic ions can lead to production of high-energy neutrinos and gamma rays through interaction with thermal radiation from the hot neutron star or the heated inner boundary region of the stellar envelope as the result of the deposition of energy by the magnetar wind. The detectability of the neutrino flux by a kilometre-scale neutrino detector such as the planned IceCube neutrino observatory is discussed.
\end{abstract}

Keywords: neutrinos — gamma rays: theory — radiation mechanisms: non-thermal

\section{Introduction}

Fast spinning magnetars have been recently proposed as potential sources for high-energy cosmic rays (e.g. Arons 2003) and as an alternative model for cosmic gamma-ray bursts (e.g. Usov 1994; Blackman \& Yi 1998; Wheeler et al. 2000; Rees \& Mészáros 2000). Fast spinning magnetars may be born from an accretioninduced collapse, in which the magnetic field is amplified exponentially to $10^{15}-10^{16} \mathrm{G}$ by dynamo processes (Dar et al. 1992; Thompson 1994). For example, such dynamo amplification may be driven by the convective neutrino heat flux (e.g. Duncan \& Thompson 1992). Magnetars with a magnetic field $B \sim 10^{15} \mathrm{G}$ and a period $P \sim 1 \mathrm{~ms}$ spin down rapidly, producing powerful transient electromagnetic radiation within a typical spindown time $\tau_{\mathrm{d}} \sim I \Omega^{2} / 2 L_{\mathrm{E}} \approx 2 \times 10^{3} \mathrm{~s}$, where $\Omega=2 \pi / P$, $I \approx 10^{45} \mathrm{~g} \mathrm{~cm}^{2}$ is the inertial moment of the pulsar, and $L_{\mathrm{E}} \sim 10^{49} \mathrm{erg} \mathrm{s}^{-1}$ is the spin-down luminosity due to the magnetic dipole radiation. Such millisecond magnetars (MSMs) should be a strong source of high-energy gamma rays and neutrinos in the initial phase of their spin-down, which is potentially observable.

In this paper, neutrinos and gamma rays from photopion production in the MSM magnetosphere and wind are discussed. Neutrinos from the magnetosphere of slowly rotating magnetars have been recently considered (Zhang et al. 2003). However, proton acceleration by the rotationinduced electric field in such slow rotators is unlikely as powerful as a normal high-field young pulsar. The typical spin-down power of a slowly rotating magnetar with $P \sim 8 \mathrm{~s}$ and $B \sim 10^{15} \mathrm{G}$ is about $10^{33} \mathrm{erg} \mathrm{s}^{-1}$, much less than that of the Crab pulsar $\left(2 \times 10^{38} \mathrm{erg} \mathrm{s}^{-1}\right)$. Their prediction of the neutrino flux is only marginal for a square-kilometre neutrino detector. As a typical MSM has the initial spin-down power of $10^{10}-10^{11}$ times that of the Crab pulsar, neutrinos from these exotic objects can be potentially observable even at an extragalactic distance by a kilometre-scale detector (Luo 2005).

A neutron star forms with a hot surface on which free emission of charged particles occurs. For pulsars with an obtuse inclination angle (that is, the angle between the magnetic axis and the rotation axis is $>\pi / 2$ ), the outflowing primary particles are ions. The physical conditions on the surface of a strongly magnetised neutron star are not well constrained by the current observations (e.g. Romani 1987), let alone the surface conditions of MSMs. Although the surface of a new-born neutron star may be rich in iron nuclei, heavy nuclei may be partially or completely photodisintegrated in the strong radiation field and the dominant components of the outflow are those much lighter nuclei such as helium or protons. Here, one assumes the outflows are protons that are accelerated to ultra-high energies. These relativistic protons can interact with photons from the thermal radiation originating from the neutron star or from the boundary region where the relativistic pulsar wind interacts with the stellar envelope or stellar wind. Proton-photon interaction leads to production of pions that decay to neutrinos and gamma rays. Apart from photopion production, accelerated protons may produce pions through $p p$ interactions in the remnant shell, which has been recently discussed by several authors (e.g. Blasi, Epstein, \& Olinto 2000; Nagataki 2004) and is not considered here. The relevant neutrino fluxes from photopion production are estimated and the detectability by kilometre-scale neutrino detectors is discussed. 


\section{Acceleration of Protons}

There are two distinct regions where particle acceleration occurs: inside the pulsar magnetosphere where charged particles are accelerated to ultra-relativistic energies in a rotation-induced potential, and in the pulsar wind where different acceleration mechanisms may operate. This latter case is discussed in Section 3. The magnetospheric acceleration is essentially the same as that which operates in normal pulsars. The accelerating potential arises from deviation of the charge density of outflowing particles from the Goldreich-Julian density $\rho_{\mathrm{GJ}} \approx B / 2 c P$, the density required for the corotation of charged particles with the star. The maximum potential available in MSMs, $\phi_{\mathrm{m}}=\theta_{\mathrm{d}}^{4} B R_{0} / 2$, is enormous, about $10^{22} \mathrm{~V}$ for $B=10^{15} \mathrm{G}$ and $P=1 \mathrm{~ms}$ (where $\theta_{\mathrm{d}}=\left(2 \pi R_{0} / c P\right)^{1 / 2}$ is the half-opening angle of the polar cap (PC), $R_{0}=10^{6} \mathrm{~cm}$ is the star's radius). As in a normal pulsar, due to electronpositron pair creation, only a very small fraction of the potential goes into particle acceleration. A relativistic proton can produce pairs on the thermal radiation through the interaction of thermal photons with the proton's Coulomb field, providing seed electrons. A small fraction of these electrons turn back and are accelerated downward to initiate a cascade producing copious pairs near the star and the positrons from the cascade are accelerated outward. The proton acceleration is then constrained due to the electric field screening as the result of a pair cascade by these outflowing relativistic positrons. If the electric field screening is not effective, a large returning electron flux ensues and the electric field would reverse its sign. Thus, the acceleration and pair cascade may be nonstationary with the efficiency limited by the pair production due to accelerated electrons/positrons.

The luminosity of the accelerated particles can be derived by assuming that the potential is a quadratic function of the distance $s$ (in $R_{0}$ ) to the $\mathrm{PC}, \phi=\phi_{\mathrm{m}} \epsilon s^{2}$, provided that acceleration is confined to the region very close the polar cap $s<\theta_{\mathrm{d}}$, where $\epsilon$ is the parameter that characterises the geometry effects such as the inclination angle between the rotation axis and the magnetic pole, the latitudes of the open field lines (e.g. Arons \& Scharlemann 1979), and the general relativity effects such as the frame-dragging (e.g. Harding \& Muslimov 1998). In the following, one assumes $\epsilon=1$. The potential limited by pair creation can be written in the form $\phi_{\mathrm{f}}=\phi_{\mathrm{m}} \epsilon s_{\mathrm{f}}^{2}$, where $s_{\mathrm{f}}$ is the characteristic distance (in $R_{0}$ ) at which one pair is produced per primary particle. If the acceleration region is limited to $s \leq s_{\mathrm{f}}$, the particle luminosity can be written as $L_{\mathrm{p}}=\eta L_{\mathrm{E}}$ with $\eta=\phi_{\mathrm{f}} / \phi_{\mathrm{m}} \approx s_{\mathrm{f}}^{2} \ll 1$.

The characteristic length $s_{\mathrm{f}}$ can be estimated as follows. In a superstrong magnetic field $B \gg B_{c}=m_{\mathrm{e}}^{2} c^{3} / \hbar e \approx$ $4.4 \times 10^{13} \mathrm{G}$ (the critical field at which the cyclotron energy equals the electron rest energy $m_{\mathrm{e}} c^{2}$ ), the probability for single photon decay into an electron-positron pair can be approximated by the step function, i.e. one pair is produced when the photon energy reaches the threshold (e.g. Harding, Baring, \& Gonthier 1997):
$E_{\gamma}>2 m_{\mathrm{e}} c^{2} / \sin \theta_{k B}$ for the $\|$-polarised photon and $E_{\gamma}>m_{\mathrm{e}} c^{2}\left(2 \varepsilon_{B}\right)^{1 / 2} / \sin \theta_{k B}$ for the $\perp$-polarised photon, where $\varepsilon_{B}=B / B_{c} \gg 1$, the $\perp$ and $\|$ modes correspond respectively to a photon with polarization perpendicular to and in the plane defined by the wave vector and the magnetic field, and $\theta_{k B}$ is the propagation angle with respect to the magnetic field line direction. Since the $\perp$ photon can split into low energy photons, only the first threshold condition is relevant. Two competing radiation processes, resonant inverse Compton scattering (RICS; Gonthier et al. 2000) and curvature radiation, provide injection of gamma rays for pair creation. Although nonresonant inverse Compton scattering may also contribute to pair creation, it is less efficient than RICS. Because of rapid acceleration and small curvature radius $R_{\mathrm{c}} \sim R_{0}$ in the MSM environment, the curvature radiation process dominates and gives rise to the limit (Q. Luo, in preparation), $\eta \approx\left(9 a_{\mathrm{c}} \alpha_{\mathrm{f}} / 2 \gamma_{\mathrm{m}}\right)^{2 / 3} \approx 10^{-8}(P / 1 \mathrm{~ms})^{5 / 3}\left(10^{15} \mathrm{G} / B\right)^{2 / 3}$, and the maximum proton energy $E_{\mathrm{p}} \sim 35 \mathrm{TeV}$, where $a_{\mathrm{c}} \leq 4 / 3 \theta_{\mathrm{d}}$ is the geometric factor due to the field line curvature, $\gamma_{\mathrm{m}}=\phi_{\mathrm{m}} / m_{\mathrm{e}} c^{2}$, and $\alpha_{\mathrm{f}} \approx 1 / 137$ is the fine structure constant.

\section{Magnetar Winds}

The outflows from the pulsar form a wind that is dominated by Poynting flux near the light cylinder (LC, the radius at which the rotation speed equals $c$ ). Observations of the Crab nebula provide strong evidence that the pulsar wind at the termination shock is predominantly in particle kinetic energy (Kennel \& Coroniti 1984). This leads to the conclusion that a large fraction of the Poynting flux must be converted to kinetic energy before the termination shock. The specific processes that are responsible for such conversion are not well understood (e.g. Arons 2003; Melatos 2005) and it is possible that several mechanisms may operate. If one assumes that the magnetar wind has the properties very similar to that of the normal young pulsar, the conversion of the Poynting flux to the particle kinetic energy must also occur in the magnetar wind (Arons 2003). One may express the particle luminosity as the fraction of the spin-down luminosity, $L_{\mathrm{p}}=\eta_{\mathrm{W}} L_{\mathrm{E}}$ with $\eta \ll \eta_{\mathrm{W}} \leq 1$ for young pulsars. If protons are dominant in the inertia of the wind, the maximum Lorentz factor of protons is then $\gamma=\eta_{\mathrm{W}} L_{\mathrm{E}} /\left(4 \pi c R_{\mathrm{LC}}^{2} n_{0} m_{\mathrm{p}} c^{2}\right)$, where $n_{0}$ is the proton number density at the LC.

The proton energy is limited by pair production and energy loss due to inverse Compton scattering (IC) on thermal radiation. The effect of the latter process strongly depends on the distribution of thermal radiation and also on whether the acceleration is fast enough to overcome the energy loss due to IC. In general, IC on thermal radiation from the neutron star is not important in constraining the proton energy as the photon number density is scaled as proportional to $\left(R_{0} / r\right)$ with $r \gg R_{0}$ in the wind zone. However, if acceleration is gradual and occurs in the region that is filled with diffuse thermal radiation 
(Beall \& Bednarek 2002), IC may limit the maximum proton energy. For example, for the 'surf-riding' acceleration mechanism, which is applicable to an aligned rotator (e.g. Contopoulos \& Kazanas 2002; Arons 2003), the Lorentz factor of protons increases linearly with the radial distance, $\gamma \sim r / R_{\mathrm{LC}}$. Consider thermal photons are uniformly emitted from the hot inner surface of the shell. One may assume that the thermal photon distribution is isotropic and derive the maximum Lorentz factor in the Thomson regime where the photon energy in the proton rest frame is less than the proton rest energy. The energy loss rate due to IC is given by $-\mathrm{d} \gamma / \mathrm{d} t=c \sigma_{\mathrm{T}} U_{\mathrm{ph}} \gamma^{2} / \mu_{\mathrm{p}}^{2}$, where $U_{\mathrm{ph}} \approx 7.6 \times 10^{5}\left(T / 10^{5} \mathrm{~K}\right)^{4} \mathrm{erg} \mathrm{cm}^{-3}$ is the energy density of the thermal radiation, $\sigma_{\mathrm{T}} \approx 6.65 \times 10^{-25} \mathrm{~cm}^{2}$ is the Thomson cross section, and $\mu_{\mathrm{p}}=m_{\mathrm{p}} / m_{\mathrm{e}}$ is the ratio of the proton to electron mass. The maximum Lorentz factor is obtained as $\gamma_{\mathrm{IC}} \approx 3.8 \times 10^{7}(1 \mathrm{~ms} / P)^{1 / 2}\left(10^{5} \mathrm{~K} / T\right)^{2}$. The corresponding radial distance where this limit is reached is estimated to be $r_{\mathrm{c}} \approx \gamma_{\mathrm{IC}} R_{\mathrm{LC}} \approx 1.8 \times 10^{14} \mathrm{~cm}$ for the above nominated parameters. Note that the isotropy assumption for the thermal radiation may not be appropriate if the interaction region between the wind and shell is clumpy or fragmentary, e.g. as a result of Kelvin-Helmholz instabilities. In this case, the constraint on the maximum proton energy by Compton drag may be significantly reduced.

\section{Neutrino Flux Prediction}

The dominant channel for neutrino production is photomeson production at the $\Delta$ resonance (Stecker et al. 1991; Waxman \& Bahcall 1997), $\mathrm{p}+\gamma \rightarrow \Delta \rightarrow \mathrm{n}+\pi^{+}$. As neutrons have a relatively long decay time, $\sim 10^{3} \gamma_{\mathrm{p}} \mathrm{s}$ in the observer's frame, they may decay outside the remnant (Protheroe, Bednarek, \& Luo 1998). The charged pion decay produces neutrinos, $\pi^{+} \rightarrow \mu^{+}+\nu_{\mu} \rightarrow \mathrm{e}^{+}+\nu_{\mathrm{e}}+$ $\bar{v}_{\mu}+v_{\mu}$. The cross section is peaked $\sigma_{\mathrm{p} \gamma} \approx 5 \times 10^{-28} \mathrm{~cm}^{2}$ at $E_{*} \approx 0.35 \mathrm{GeV}$ with the width $\Delta E_{*} \approx 0.12 \mathrm{GeV}$ in the proton rest frame (Stecker et al. 1991). The Lorentz factor $\gamma_{*}=1 /\left(1-\beta_{*}^{2}\right)^{1 / 2}$ of the proton for which the cross section for pion production peaks is $\gamma_{*}=E_{*} /\left[\left(1-\beta_{*} \cos \theta\right) E_{\gamma}\right]$, where $\theta$ is the photon propagation angle and $E_{\gamma}$ is the target photon energy. For $E_{\gamma} \sim k_{\mathrm{B}} T=10 \mathrm{eV}$ (where $k_{\mathrm{B}}$ is Boltzmann's constant), one has $\gamma_{*} \approx 1.6 \times 10^{7}\left(10^{5} \mathrm{~K} / T\right) /\left(1-\beta_{*} \cos \theta\right)$. Photomeson processes also produce neutral pions $\left(\pi^{0}\right)$ that can decay into $\gamma$ rays, $p+\gamma \rightarrow \Delta \rightarrow p+\pi^{0} \rightarrow p+2 \gamma$. In general, the photomeson processes are more efficient in producing $\gamma$ rays than neutrinos.

In the magnetosphere, the main source for the target photons is thermal radiation from the neutron star's hot surface. The head-on scattering photons (in the proton's rest frame) with $\cos \theta \sim\left[(s+1)^{2}-1\right]^{1 / 2} /(s+1) \sim$ $(2 s)^{1 / 2} \ll 1$ contribute the most to pion production. It can be shown that the proton Lorentz factor can satisfy the resonance condition provided that the surface temperature is $T \sim 10^{7}-10^{8} \mathrm{~K}$. At $P=1 \mathrm{~ms}$, the maximum Lorentz factor is $3.5 \times 10^{4}$ and $T \geq 5 \times 10^{7} \mathrm{~K}$ is needed. This condition can easily be satisfied for a new-born neutron star. With the photon number density $n_{\mathrm{ph}} \sim 4 \times 10^{25} \mathrm{~cm}^{-3}$, the characteristic length for pion production is $s_{\pi} \sim 1 / n_{\mathrm{ph}} \sigma_{\mathrm{p} \gamma} \sim 50 \mathrm{~cm}$. The typical pion energy is $E_{\pi} \sim 0.2 E_{p}$. Pions decay on a time $10^{-8} E_{\pi} / m_{\pi} c^{2} \mathrm{~s}$ in the observer's frame and may suffer significant cooling due to IC (e.g. Beall \& Bednarek 2002; Zhang et al. 2003). Although some of the charged pions may be produced at $s<s_{\mathrm{f}}$ and can be re-accelerated, most pions would decay outside the acceleration region $s>s_{\mathrm{f}}$ and their final energy should be determined by the cooling. By equating the decay time to the cooling time, one obtains the IC limited pion energy $E_{\pi}=10^{2}\left(5 \times 10^{7} \mathrm{~K} / T\right)^{2} \mathrm{GeV}$ and neutrino energy $E_{\nu}=E_{\pi} / 4=25 \mathrm{GeV}$, well below the threshold of large neutrino detectors like the IceCube. The typical neutrino energy can be higher than the above estimate if proton acceleration or re-acceleration of charged pions occurs in a high altitude range extending to the LC (e.g. Arons 1981; Cheng, Ho, \& Ruderman 1986; Romani 1996) where the photon number density is reduced and pion production occurs at a higher threshold. The neutrino flux is limited by the luminosity of protons, which is only an $\eta \sim 10^{-8}$ fraction of the total spin-down power $\left(10^{49}-10^{50} \mathrm{erg} \mathrm{s}^{-1}\right)$. The maximum neutrino luminosity is less than $5 \%$ of the proton luminosity if there is no significant re-acceleration of protons or multiple pion production. Thus, neutrinos originating from the inner magnetosphere have a luminosity $\leq 5 \times 10^{40} \mathrm{erg} \mathrm{s}^{-1}$.

Neutral pion decay can produce $\mathrm{TeV}$ photons in the magnetosphere. In the superstrong magnetic field, the $\perp$ polarised photons can split into photons with a much lower energy. There is no splitting of the $\|$-polarised photons, but they can decay into a electron-positron pair in the ground Landau state (Weise \& Melrose 2002). Thus, most of these $\mathrm{TeV}$ photons are reprocessed to photons with energies below the pair production threshold ( $\mathrm{MeV}$ energy).

Efficient conversion of the spin-down power to particle kinetic energy occurs in the pulsar wind. This opens up the possibility of photomeson production by accelerated protons in the pulsar wind on thermal radiation (e.g. Beall \& Bednarek 2002; Granot \& Guetta 2003). The target photons can be provided by the thermal radiation from the interaction region where the pulsar wind terminates at the expanding stellar envelope or stellar wind. Alternatively, the target photons can originate from the hot ejecta in the early phase of the newly formed pulsar, as suggested by Beall \& Bednarek (2002). Assuming that a fraction $\xi_{\mathrm{W}}$ of the spin-down power is intercepted by the boundary region, the effective temperature is estimated as (e.g. Rees \& Mészáros 2000) $T \approx\left(\xi_{\mathrm{W}} L_{\mathrm{E}} / 4 \pi \sigma_{\mathrm{SB}} r_{\mathrm{b}}^{2}\right)^{1 / 4} \approx 10^{5} \mathrm{~K}$ for $\xi_{\mathrm{W}} L_{\mathrm{E}}=10^{47} \mathrm{erg} \mathrm{s}^{-1}$ and $r_{\mathrm{b}}=10^{14} \mathrm{~cm}$, where one expresses the thermal luminosity as a fraction $\xi_{\mathrm{W}}$ of the spin-down power, $\sigma_{\mathrm{SB}}$ is Stefan-Boltzmann's constant, and $r_{\mathrm{b}}$ is the radial distance from the star's center to the interface between the pulsar wind and the expanding remnant shell. The temperature decreases as the shell expands at a speed of about $v_{\mathrm{b}}=2000 \mathrm{~km} \mathrm{~s}^{-1}$. 
The neutrino flux can be estimated from $F_{v} \approx \eta_{\mathrm{W}} \eta_{\nu}$ $L_{\mathrm{E}} /\left(\Delta \Omega D^{2} E_{v}\right)$, where $D$ is the distance, $\Delta \Omega$ is the beaming solid angle, $E_{\nu}$ is the typical neutrino energy, and $\eta_{\nu}$ is the fraction of proton energy going into neutrinos. One has $\eta_{\nu} \sim 0.05$ for a single pion production and $\eta \sim 1 / 3$ for multiple pion production. I assume that pion production occurs in the inner region of the remnant shell due to head-on colliding of the relativistic protons in the wind with the thermal photons. The neutrino energy is limited by energy loss due to IC to $E_{\nu} \sim E_{\pi} / 4 \sim 10^{3} \mathrm{TeV} \ll 0.2 E_{p}$. The flux can reach $3 \times 10^{-12} \mathrm{~cm}^{-2} \mathrm{~s}^{-1}$ for $D=10 \mathrm{Mpc}$. One may compare this flux with that from the atmospheric background with a spectrum $\phi_{v}^{\mathrm{b}} \sim 10^{-7}\left(E_{v} / 1 \mathrm{TeV}\right)^{-2.5} \mathrm{~cm}^{-2} \mathrm{~s}^{-1} \mathrm{sr}^{-1}$. Since the IceCube will have an angular resolution less than $1^{\circ}$, the background flux from this small circular patch is $F_{v}^{\mathrm{b}} \approx \phi_{\nu}^{\mathrm{b}}(\pi / 180)^{2} \approx 2.6 \times 10^{-14} \mathrm{~cm}^{-2} \mathrm{~s}^{-1}$ for $E_{\nu}=10 \mathrm{TeV}$. The predicted initial neutrino flux is well above this background flux. The probability of the muon event in the ice for neutrinos with above $\mathrm{TeV}$ energy is about $P_{\nu \mu} \approx 2 \times 10^{-6}\left(E_{\nu} / 1 \mathrm{TeV}\right)$ (Halzen \& Hooper 2002). The corresponding event rate is

$$
\begin{aligned}
N_{v} \approx & \frac{\eta_{\mathrm{W}} \eta_{\nu} L_{\mathrm{E}} P_{\nu \mu}}{\Delta \Omega D^{2} E_{\nu}} \\
\approx & 2 \times 10^{3}\left(\frac{\eta_{\mathrm{W}}}{10^{-5}}\right)\left(\frac{4 \pi}{\Delta \Omega}\right)\left(\frac{L_{\mathrm{E}}}{10^{49} \mathrm{erg} \mathrm{s}^{-1}}\right) \\
& \times\left(\frac{D}{10 \mathrm{Mpc}}\right)^{-2} \mathrm{~km}^{-2} \mathrm{yr}^{-1} .
\end{aligned}
$$

The neutrino flux can be boosted significantly if the emission is beamed within an angular range $\Delta \Omega_{0}<1$.

The light curve is characterised by a rapid increase in the flux density to a peak, followed by decay as the MSM spins down with the remnant expanding away. Assuming the pulsar spins down through dipole radiation, it takes about ten days for the MSM to spin down to a period $P \sim 21 \mathrm{~ms}$ (for $B=10^{15} \mathrm{G}$ ). The spin-down power is decreased by five orders of magnitude to $L_{\mathrm{E}} \sim 10^{44} \mathrm{erg} \mathrm{s}^{-1}$. The event rate averaged over a duration $\Delta t$ can be written as $\bar{N}_{\mu} \approx N_{\mu} \tau_{\mathrm{d}} / \Delta t$, where the pulsar period is written in the form $P=P_{0}\left(1+t / \tau_{\mathrm{d}}\right)^{1 / 2}, P_{0}=1 \mathrm{~ms}$ is the initial period, and $\tau_{\mathrm{d}}=3 c^{3} I P_{0}^{2} /\left(4 \pi^{2} B^{2} R_{0}^{2}\right) \approx 2 \times 10^{3} \mathrm{~s}$ is the time for the period to increase by a factor of $2^{1 / 2}$. The thermal radiation may be very inhomogeneous, concentrated near the interaction region between the wind and the remnant shell. This permits a much higher $\eta_{\mathrm{W}}$. Note that in the Crab nebula, one deduces $\eta_{\mathrm{W}} \sim 0.1-0.2$ (Gaensler et al. 2002). If one assumes that $\eta_{\mathrm{W}} \sim 10^{-2}$ of the total spindown power goes into proton acceleration, one estimates $\bar{N}_{\mu} \sim 10^{2}\left(\eta_{\mathrm{W}} / 10^{-2}\right)(1 \mathrm{~d} / \Delta t)$ events per day during $\Delta t$ for $D=10 \mathrm{Mpc}$. As the rate drops off proportional to $1 / \Delta t$, one would see at least a total of $10^{2}$ events.

\section{Conclusions}

Fast spinning magnetars can be a strong source of highenergy neutrinos and gamma rays in the early phase of their spin down. Like normal pulsars, strong particle acceleration in the magnetosphere occurs. Accelerated protons can interact with photons from the surface thermal emission producing pions. Neutrinos can be obtained from pion decay. However, in the superstrong magnetic field, pair production constrains the acceleration efficiency to $\eta \sim 10^{-8}$. Therefore, the neutrino luminosity is severely constrained to less than $10^{-8}$ of the total spin-down power. Furthermore, the pion cooling due to IC limits the neutrino energy to about $25 \mathrm{GeV}$, well below the designed threshold of the future IceCube. The maximum neutrino energy may be considerably higher than the estimate derived here if acceleration extends to high altitudes such as slot gap models (e.g. Arons 1981) or if re-acceleration of charged pions occurs near the LC where the constraint by IC is less severe than near the PC.

Neutrinos may be produced in photonmeson processes in the relativistic magnetar wind. Protons accelerated in the wind can produce pions on the target photons from thermal radiation in the regions where the magnetar wind interacts with the ejecta or stellar wind. Since a large fraction of the spin-down power may be converted to proton energy, the neutrino luminosity can be significantly higher than that from the magnetosphere. Since the energy loss of charged pions due to IC on the thermal radiation in the wind is less severe than in the magnetosphere, the maximum neutrino energy can reach $10^{3} \mathrm{TeV}$, well above the threshold of the IceCube. The estimated event rate is well above the detection level.

The model discussed here is similar to that proposed by Beall \& Bednarek (2002) in that photomeson production in the relativistic pulsar wind is the main channel for neutrinos. However, in their model, which is applied to normal young pulsars, particle acceleration is assumed to occur rapidly near the LC in the relativistic pulsar wind. There is no detailed theory for acceleration near the $\mathrm{LC}$; recent work on acceleration in the pulsar relativistic wind suggests that acceleration occurs further away from the LC (e.g. Lyubarsky \& Kirk 2001; Contopoulos \& Kazanas 2002; Melatos 2003). In the Contopoulos \& Kazanas (2002) model for an aligned rotator, the plasma in the wind is accelerated linearly with the radial distance. Such an acceleration mechanism is also used in the magnetar model for high-energy cosmic ray sources proposed recently by Arons (2003). It is suggested here that in the early phase of spin-down, if the region inside the remnant shell is filled with a diffuse thermal radiation field, IC would constrain any gradual acceleration. It is shown that the IC-limited Lorentz factor of protons can still satisfy the threshold for pion production through the $\Delta$ resonance.

\section{Acknowledgements}

The author thanks Don Melrose for helpful comments.

\section{References}

Arons, J. 1981, ApJ, 248, 1099

Arons, J. 2003, ApJ, 589, 871

Arons, J., \& Scharlemann, E. 1979, ApJ, 231, 854 
Beall, J. H., \& Bednarek, W. 2002, ApJ, 569, 343

Blackman, E., \& Yi, I. 1998, ApJ, 498, L31

Blasi, P., Epstein, R. I., \& Olinto, A. V. 2000, ApJ, 533, L123

Cheng, K. S., Ho, C., \& Ruderman, M. 1986, ApJ, 300, 500

Contopoulos, I., \& Kazanas, D. 2002, ApJ, 566, 336

Dar, A., Kozlovsky, B., Nussinov, S., \& Ramaty, R. 1992, ApJ, 388, 164

Duncan, R., \& Thompson, C. 1992, ApJ, 392, L9

Gaensler, B. M., et al. 2002, ApJ, 569, 878

Gonthier, P., Harding, A., Baring, M., Costello, R., \& Mercer, C. 2000, ApJ, 540, 907

Granot, J., \& Guetta, A. 2003, PhRvL, 90, 191102

Halzen, F., \& Hooper, D. 2002, RPPh, 65, 1025

Harding, A., \& Muslimov, A. 1998, ApJ, 508, 328

Harding, A., Baring, M., \& Gonthier, P. 1997, ApJ, 476, 246

Kennel, C. F., \& Coroniti, F. V. 1984, ApJ, 283, 694

Luo, Q. 2005, AIP proceedings, in press
Lyubarsky, Y., \& Kirk, J. 2001, ApJ, 547, 437

Melatos, A. 2005, in Highlights of Astronomy, Vol. 13, ed O. Engvold (San Francisco: ASP), in press

Nagataki, S. 2004, ApJ, 600, 883

Protheroe, R. J., Bednarek, W., \& Luo, Q. 1998, APh, 9, 1

Rees, M., \& Mészáros, P. 2000, ApJ, 545, L73

Romani, R. 1987, ApJ, 313, 718

Romani, R. 1996, ApJ, 470, 469

Stecker, F., Done, C., Salamon, M. H., \& Sommers, P. 1991, PhRvL, 66, 2697

Thompson, C. 1994, MNRAS, 270, 480

Usov, V. 1994, MNRAS, 267, 1035

Waxman, E., \& Bahcall, J. N. 1997, PhRvL, 78, 2292

Weise, J., \& Melrose, D. B. 2002, MNRAS, 329, 115

Wheeler, J. C., Yi, I., Höflich, P., \& Wang, L. 2000, ApJ, 537, 810

Zhang, B., et al. 2003, ApJ, 595, 346 